\title{
Article
}

\section{Development of an Adsorbent for Cs Removal Synthesized by Radiation-Induced Graft Polymerization}

\author{
Takuya SHIBATA*, Noriaki SEKO, Haruyo AMADA, Noboru KASAI, \\ Seiichi SAIKI, Hiroyuki HOSHINA and Yuji UEKI
}

Environmental Polymer Group, Quantum Beam Science Center, Sector of Nuclear Science Research, Japan Atomic Energy Agency, 1233 Watanuki, Takasaki, Gunma 370-1292, Japan

(Manuscript received June 17, 2014; accepted August 7, 2014)

\begin{abstract}
One of the most important tasks for recovering living area contaminated by the accident at the Fukushima Daiichi Nuclear Power Station is to secure a safe water supply. Therefore, we developed a Cs adsorbent wherein ammonium 12-molybdophosphate (AMP) was loaded by radiation-induced graft polymerization on a fibrous trunk material and crosslinked to form a structure wherein the loaded AMP is stable. To optimize the synthesis of this Cs adsorbent, a $9 \mathrm{G}$ (G: dimethacrylate) polyethylene glycol dimethacrylate (PEGDM) was used as a crosslinking reagent and the adding volume was adjusted to $1 \mathrm{~mol} \%$ in a monomer solution to synthesize the Cs adsorbent with a $200 \%$ degree of grafting. Our evaluation of the adsorption performance of the Cs adsorbent indicates that it can be applied to a standard $\mathrm{pH}$ range for drinking water (from $\mathrm{pH} 5.8$ to 8.6) and captures sufficient $\mathrm{Cs}$, even in a solution containing $1 \mathrm{mg} / \mathrm{L}$ of $\mathrm{K}$, $\mathrm{Ca}, \mathrm{Mg}$, and $\mathrm{Na}$ ions, to yield a $\mathrm{Cs}$ concentration comparable to that of stream water.

Keywords: Cs, Ammonium 12-molybdophosphate, Radiation-induced graft polymerization, Crosslinking
\end{abstract}

\section{Introduction}

Securing safe water is one of the most important tasks to decontaminate living area contaminated by the 2011 accident at the Fukushima Daiichi Nuclear Power Station. Decontamination activities have been ongoing for more than 3 years. Although radioactive $\mathrm{Cs}$, such as ${ }^{134} \mathrm{Cs}$ and ${ }^{137} \mathrm{Cs}$, has only barely been detected in environmental water, the results of monitoring stream water in Fukushima Prefecture by the Ministry of Environment in Japan reveal radioactive $\mathrm{Cs}$ in 7 out of 700 samples collected from December 2013 to February 2014 ${ }^{1)}$, and all samples containing Cs had Cs concentrations lower than the limit of 10 $\mathrm{Bq} / \mathrm{L}$ set as the guideline for Welfare drinking water by The Ministry of Health, Labour in Japan ${ }^{2)}$. Disaster victims have demanded the development of decontamination methods able to reduce $\mathrm{Cs}$ concentrations to even lower level.
Various types of Cs adsorbents have been investigated, such as an zeolite ${ }^{3)}$, ferrocyanide ${ }^{4)}$, crown ether ${ }^{5)}$, and ammonium 12-molybdophosphate $(\mathrm{AMP})^{6)}$. Although AMP is known to have a high affinity for Cs ions, it has never been used as a Cs adsorbent in the form of AMP fixed in the powdered state in a resin. Although the AMP adsorbent works well, it has poor stability in some resins during $\mathrm{Cs}$ adsorption.

In this study, we developed a new $\mathrm{Cs}$ adsorbent, in which we introduced a crosslinked structure into the grafted AMP chains to ensure a highly stable Cs adsorbent. We found the most suitable crosslinking regent and its concentration. In addition, the optimized adsorbent was evaluated for its application to drinking water.

\footnotetext{
* Corresponding author. Fax: +81273469381

E-mail: shibata.takuya@jaea.go.jp
} 


\section{Experiment}

\subsection{Materials and Chemicals}

We used a nonwoven fabric (NF) made by polyethylene (grade EX02, Kurashiki Textile Manufacturing Co., Ltd.) as the fibrous trunk material. AMP $\left[\left(\mathrm{PMo}_{12} \mathrm{O}_{40}\right)\left(\mathrm{NH}_{4}\right)_{3}\right]$ was purchased from Nippon Inorganic Colour and Chemical Co., Ltd. Glycidyl methacrylate (GMA) was purchased from Mitsubishi Gas Chemical Company, Inc. Dimethylsulfoxide (DMSO) was purchased from Kishida Chemical Co., Ltd. Polyoxethylene sorbitan monooleate (Tween 80 ), which we used as a surfactant, was purchased from Kanto Chemical Co., Ltd. As the crosslinking reagents, we used ethylene glycol dimethacrylate (1G), polyethylene glycol 200 dimethacrylate $(4 \mathrm{G})$, polyethylene glycol 400 dimethacrylate $(9 \mathrm{G})$, polyethylene glycol 600 dimethacrylate $(14 \mathrm{G})$, and polyethylene glycol 1000 dimethacrylate (23G) as the types of polyethylene glycol dimethacrylate (PEGDM) purchased from Shin-Nakamura Chemical Co., Ltd. Nitric acid, purchased from Kanto Chemical Co., Ltd. and methanol $(\mathrm{MeOH})$, purchased from Taiyo Chemical Industry Co., Ltd., were used for washing grafted NF.

For quantitative analysis in the adsorption experiment, we purchased a standard solution $(1,000 \mathrm{mg} / \mathrm{L})$, such as $\mathrm{Cs}, \mathrm{K}, \mathrm{Ca}, \mathrm{Mg}$, and $\mathrm{Na}$, from Kanto Chemical Co., Ltd. Ultrapure nitric acid and lithium hydroxide were purchased from Kanto Chemical Co., Ltd. to adjust the pH.

\subsection{Radiation-induced Graft Polymerization}

\subsubsection{Preparation of Monomer Solution}

The monomer solution was prepared by mixing the fol- lowing regents in the order in which they appear: Tween 80, GMA, PEGDM, and AMP into DMSO solvent. The weight ratio of GMA, Tween 80 , AMP, and DMSO was 10 , $0.8,0.5$, and 90 , respectively. The molar ratio of PEGDM ranged from 1 to $20 \mathrm{~mol} \%$ against the GMA concentration. After adding each reagent, the solution was mixed for 10 min using a homogenizer until dissolved and/or dispersed. The monomer solution was deoxygenated by bubbling with nitrogen gas.

\subsubsection{Synthesis of Cs Adsorbent}

Fig. 1 shows the scheme for synthesizing Cs adsorbent. The NF, cut into $4 \mathrm{~cm}$ squares, was irradiated to $50 \mathrm{kGy}$ by an electron beam in a nitrogen gas atmosphere at dry-ice temperature. The irradiated NF was grafted into the prepared monomer solution. The grafting reaction was conducted for $0.5-24$ hours at $40^{\circ} \mathrm{C}$. After grafting, the grafted $\mathrm{NF}$ was washed by the following regents in the order in which they appear: distilled water, $0.01 \mathrm{M}$ nitric acid, and $\mathrm{MeOH}$ for removing residual monomer. Next, the grafted $\mathrm{NF}$ was dried by vacuum at $30^{\circ} \mathrm{C}$. The degree of grafting (Dg) was calculated based on the weight gain resulting from grafting as follows:

Degree of grafting : Dg $[\%]=\left(W_{\mathrm{g}}-W_{0}\right) / W_{0} \times 100$

where $W_{\mathrm{g}}$ is the weight of the adsorbent after grafting, and $W_{0}$ is the weights of the NF before grafting.

\subsubsection{Batch and Column of Adsorption}

The adsorption of the synthesized AMP-loaded adsor-

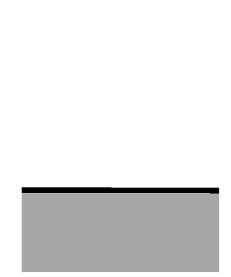

Nonwoven fabric

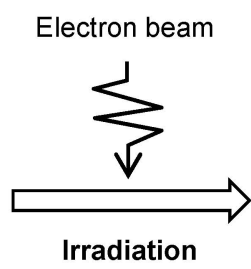

Irradiation

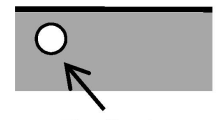

Radical
Crosslinked (PEGDM) chain
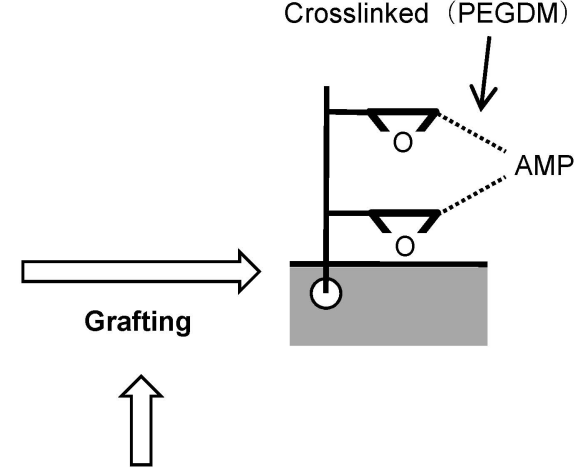

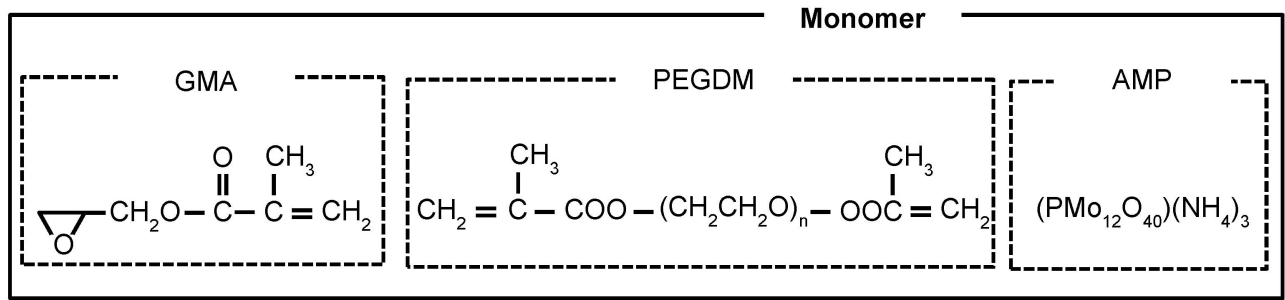

Fig. 1 Scheme for synthesis of Cs adsorbent. 
bent was evaluated by batch and column adsorption. For batch adsorption, the adsorbent was cut into $1 \mathrm{~cm}$ squares, immersed in $50 \mathrm{~mL}$ of $\mathrm{Cs}$ solution, and stirred for $24 \mathrm{~h}$. The Cs solution was prepared by diluting a standard Cs solution and adjusting the $\mathrm{pH}$. Column adsorption was performed by pumping $1 \mu \mathrm{g} / \mathrm{L}$ diluted $\mathrm{Cs}$ solution into a column at a flow rate of $1.3 \mathrm{~mL} / \mathrm{min}$ Cs solution into a column at a flow rate of $1.3 \mathrm{~mL} / \mathrm{min}$ ( $\mathrm{SV}$ : space velocity, $400 \mathrm{~h}^{-1}$ ). The circular adsorbent with a diameter of $7 \mathrm{~mm}$ and a height of $5 \mathrm{~mm}\left(0.2 \mathrm{~cm}^{3}\right)$ was packed into the column.

The metal-ion concentration was analyzed using inductively coupled plasma mass spectrometry (Seiko Instruments Inc., SPQ9700) and inductively coupled plasma optical emission spectrometry (SPECTRO Analytical Instruments GmbH., Spectro Arcos).

The stability of the loaded AMP was investigated by analyzing the molybdenum (Mo) desorbed from the adsorbent in batch adsorption, and calculated as follows:

$$
\text { Stability of loaded AMP }[-]=M_{\mathrm{N}} / M_{\mathrm{CL}}
$$

where $M_{\mathrm{N}}$ is the amount of Mo desorbed from the noncrosslinked adsorbent and $M_{\mathrm{CL}}$ is the amount of Mo desorbed from the crosslinked adsorbent. The stability of AMP loaded in the noncrosslinked adsorbent was set to unity as a benchmark. Therefore, the stability of the loaded AMP was expressed as desorbed Mo of the noncrosslinked adsorbent versus that of the crosslinked adsorbent.

\subsubsection{AMP Composition of Cs Adsorbent}

The Cs adsorbed by AMP was determined by wet digestion. The Cs adsorbent that contained AMP was completely dissolved by immersion in concentrated nitric acid and heating with microwaves. The Mo concentration in the solution was determined using inductively coupled plasma atomic emission spectroscopy (Perkin Elmer Inc., Optima DV4300). The Mo concentration was calculated as follows:

$$
\text { Loaded Mo }[\mathrm{mmol} / \mathrm{kg}-\text { absorbent }]=C V / M_{\mathrm{M}} / W
$$

where $C$ is the concentration of Mo in the nitric acid solution, $V$ is the volume of the nitric acid solution, $M_{\mathrm{M}}$ is the molecular weight of Mo (30.97), and $W$ is the weight of the Cs adsorbent.

\section{Results and Discussion}

\subsection{Optimal Synthesis of Cs Adsorbent}

We investigated several factors to develop an optimal Cs adsorbent: (1) a suitable PEGDM, (2) the concentration of clarified PEGDM in the monomer, (3) Dg. The synthe- sized adsorbents were evaluated by batch adsorption in 50 $\mu \mathrm{g} / \mathrm{L}$ of $\mathrm{Cs}$ solution at $\mathrm{pH}$ 5.6.

\subsubsection{Selection of PEGDM Type as Crosslinking Rea- gent}

To select a suitable PEGDM as the crosslinking reagent, Cs adsorbents with $\mathrm{Dg}=200 \%$ were synthesized by adding $1 \mathrm{~mol} \%$ of $1 \mathrm{G}, 4 \mathrm{G}, 9 \mathrm{G}, 14 \mathrm{G}$, and $23 \mathrm{G}$ PEGDM into the monomer for grafting, and the resulting adsorbents were investigated by batch adsorption, as shown in Fig. 2. The AMP loaded into the crosslinked adsorbent was highly stable, and the crosslinked adsorbent also exhibited a higher adsorptivity for $\mathrm{Cs}$ than the noncrosslinked adsorbent. The loaded AMP was most stable in the crosslinked adsorbent with $1 \mathrm{G}$ and $9 \mathrm{G}$. With more than $1.1 \mathrm{mmol} / \mathrm{kg}$ adsorbent), the crosslinked adsorbent with $9 \mathrm{G}$ and $14 \mathrm{G}$ adsorbed the most $\mathrm{Cs}$. The crosslinked adsorbent with $9 \mathrm{G}$ was the most effective; compared with the noncrosslinked adsorbent, the stability of the loaded AMP was 15 times better and $\mathrm{Cs}$ adsorption was 5 times higher. The length of the $9 \mathrm{G}$ chain may be most suitable for loaded AMP and Cs adsorption compared with the short $1 G$ chain, which offered low Cs affinity, and the long $14 \mathrm{G}$ and $23 \mathrm{G}$ chains, for which the loaded AMP was unstable.

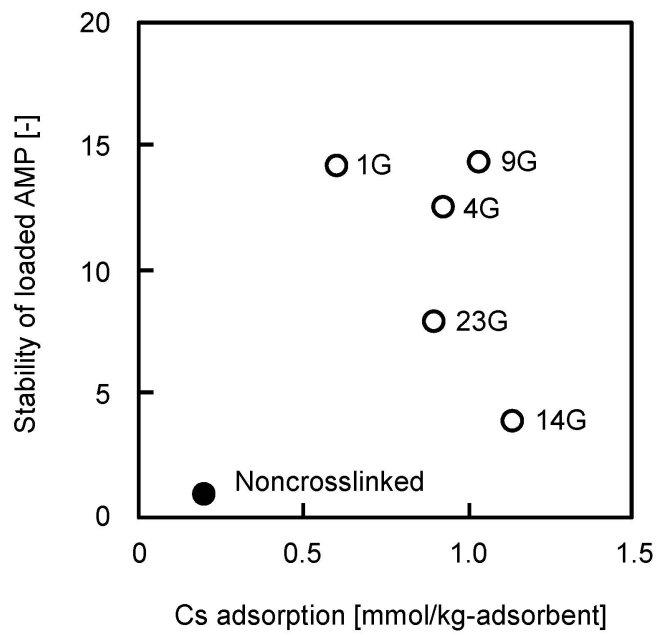

Fig. 2 Stability of loaded AMP and Cs adsorption for $\mathrm{Dg}=$ $200 \%$. The molar ratio of PEGDM to GMA is $1 \mathrm{~mol} \%$.

\subsubsection{Amount of 9G per Monomer}

To investigate the amount of $9 \mathrm{G}$ added per monomer, Cs adsorbents with $\mathrm{Dg}=200 \%$ were synthesized by adding between 1 to $20 \mathrm{~mol} \%$ of $9 \mathrm{G}$ into the monomer for grafting. The synthesized adsorbents were then investigated by batch adsorption, as shown in Fig. 3. The adsorbents synthesized with 10 and $20 \mathrm{~mol} \% 9 \mathrm{G}$ had the most stable loaded AMP. However, the Cs adsorption was 70\% less than 


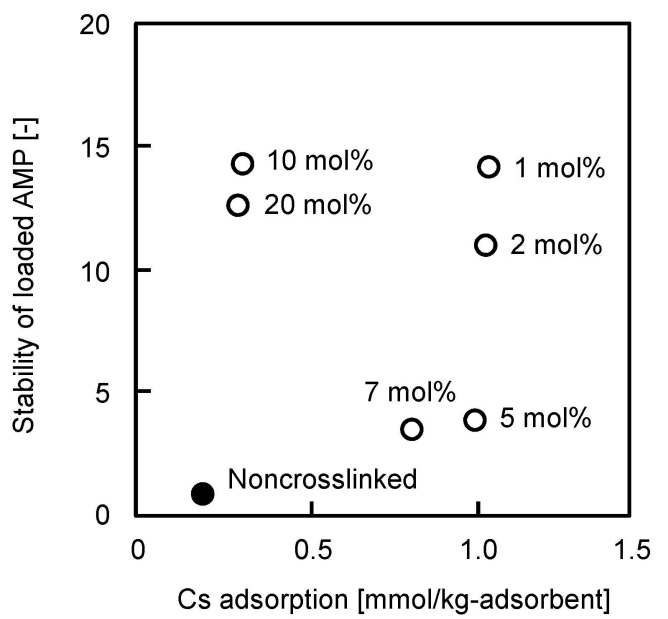

Fig. 3 Stability of loaded AMP and Cs adsorption for $\mathrm{Dg}=$ $200 \%$. The molar ratio of $9 \mathrm{G}$ to GMA varied from 1 to $20 \mathrm{~mol} \%$.

that of absorbent synthesized with 1,2 , and 5 mol\% $9 \mathrm{G}$. This result suggests that the loaded AMP was highly stable because many crosslinked chains had formed in the grafted layer. However, the adsorption of $\mathrm{Cs}$ was low because the crosslinked chains are highly hydrophobic. In terms of AMP stability and Cs adsorption, and without invoking a higher hydrophobicity, the most suitable concentration was $1 \mathrm{~mol} \%$ 9G.

\subsubsection{High Performance Dg}

To determine the best Dg for the adsorbent, Cs adsorbents from $\mathrm{Dg}=50 \%$ to $400 \%$ were crosslinked by adding $1 \mathrm{~mol} \% 9 \mathrm{G}$ to the monomer for grafting. The evolution of Dg is shown in Fig. 4. Dg increases with the reaction time. After grafting for $9 \mathrm{~h}$, Dg reached $400 \%$ and then leveled off. When synthesized at different reaction times, Dg was $50 \%$ for $0.3 \mathrm{~h}, 100 \%$ for $1 \mathrm{~h}, 200 \%$ for $2 \mathrm{~h}, 300 \%$ for $5 \mathrm{~h}$, and $400 \%$ for 24 h. Fig. 5 shows the degree of Cs adsorption as determined by batch adsorption at various Dg values, as previously shown in Fig. 4 . The degree of Cs adsorption increased with $\mathrm{Dg}$, and the degree of Cs adsorption with $\mathrm{Dg}=400 \%$ reached $100 \%$. Fig. 6 shows the Mo loaded onto adsorbent vs adsorbent weight and desorbed Mo as determined by batch adsorption for various Dg values, as previously shown in Fig. 4 . The amount of Mo loaded increased with Dg. A maximum Mo density of $33.6 \mathrm{mmol} /$ (kg-adsorbent) was obtained for $\mathrm{Dg}=400 \%$. The degree of desorbed Mo reached a maximum at 1.5 $\mathrm{mmol} /$ (kg-adsorbent) for $\mathrm{Dg}=100 \%$, and the amount of desorbed Mo for $\mathrm{Dg}=50 \%, 100 \%, 200 \%, 300 \%$, and $400 \%$ were $0.5,1.5,0.2,0.2$, and $0.3 \mathrm{mmol} /$ (kg-adsorbent), respectively. Although the amount of desorbed Mo increased in going from $\mathrm{Dg}=50 \%$ to $\mathrm{Dg}=100 \%$, it

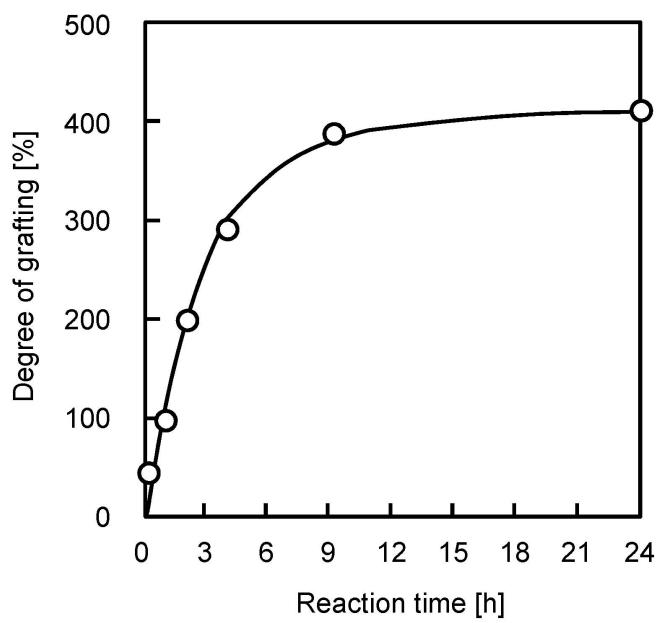

Fig. 4 Degree of grafting vs reaction time. The molar ratio of $9 \mathrm{G}$ to GMA is $1 \mathrm{~mol} \%$.

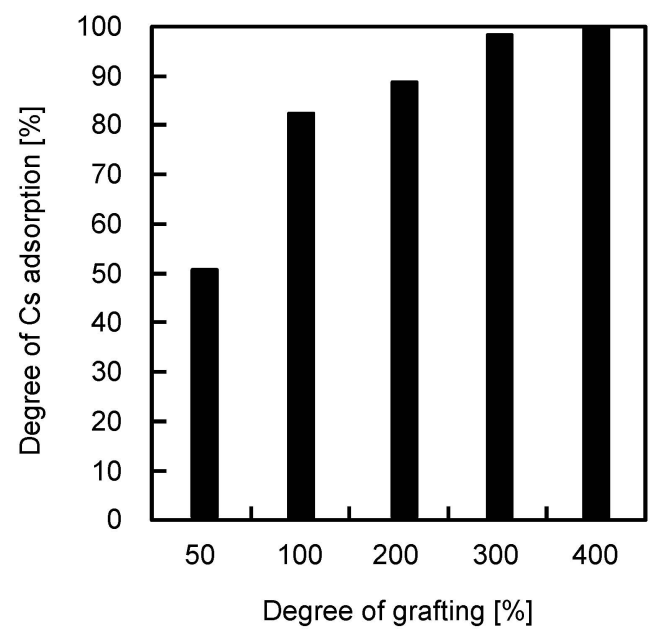

Fig. 5 Cs adsorption for various $\mathrm{Dg}$ in batch adsorption. The molar ratio of $9 \mathrm{G}$ to GMA is $1 \mathrm{~mol} \%$.

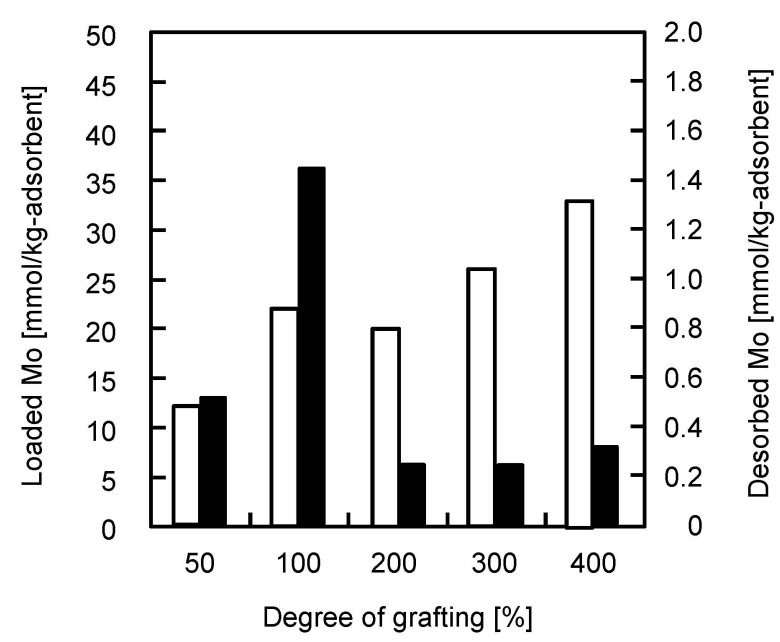

Fig. 6 Mo loaded in adsorbent and desorbed Mo in batch adsorption. The molar ratio of $9 \mathrm{G}$ to GMA is $1 \mathrm{~mol} \%$; $(\square)$ loaded Mo, ( $\square$ ) desorbed Mo. 


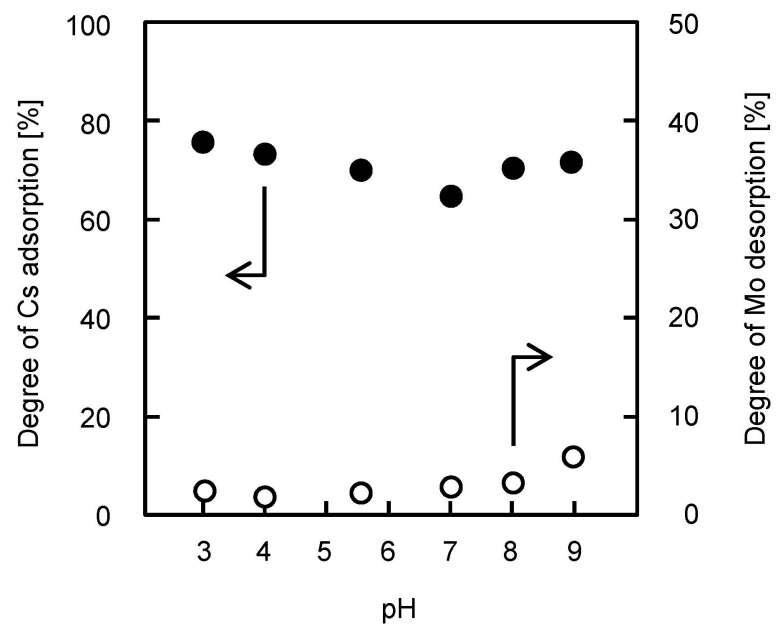

Fig. $7 \mathrm{pH}$ dependency in batch adsorption for $\mathrm{Dg}=200 \%$. The molar ratio of $9 \mathrm{G}$ to $\mathrm{GMA}$ is $1 \mathrm{~mol} \%$.

decreased significantly in going from $\mathrm{Dg}=200 \%$ to $\mathrm{Dg}=$ $400 \%$. In terms of $\mathrm{Cs}$ adsorption and the stability of the loaded AMP, $\mathrm{Dg}=200 \%$ was optimum for obtaining a high-performance adsorbent.

\subsection{Characteristics of Cs Adsorption}

For practical use in decontamination, the $\mathrm{pH}$ dependency and $\mathrm{Cs}$ selectivity as a function of coexisting ions are important to understand. The efficiency of the optimized adsorbent was evaluated by using it with environmental water. The $\mathrm{pH}$ dependency was determined by batch adsorption using a $50 \mu \mathrm{g} / \mathrm{L}$ Cs solution with the $\mathrm{pH}$ ranging from 3.0 to 9.0. Cs selectivity was investigated by using a $1 \mu \mathrm{g} / \mathrm{L} \mathrm{Cs}$ solution adjusted to $\mathrm{pH} 6.0$ and coexistence was determined using $1 \mathrm{mg} / \mathrm{L}$ of $\mathrm{K}, \mathrm{Ca}, \mathrm{Mg}$, and $\mathrm{Na}$ ions by column adsorption.

Fig. 7 shows the pH dependency as determined by batch adsorption for adaptivity to various contaminated water The degrees of $\mathrm{Cs}$ adsorption in all ranges of $\mathrm{pH}$ were over $60 \%$. Although desorbed Mo at pH 9.0 was $6 \%$, which was higher than that for $\mathrm{pH}$ ranging from 3.0 to 8.0 , the desorbed Mo for $\mathrm{pH}$ ranging from 3.0 to 8.0 could be reduced to less than $3 \%$. These results satisfy the requirements for drinking water because the acceptable $\mathrm{pH}$ value was between 5.8 and 8.6, as per the drinking water standard. Therefore, the proposed graft adsorbent may satisfy the Food Sanitation Act, contingent upon inspection by Japan Food Research Laboratories as a third-party organization. To evaluate the practical use of the proposed adsorbent, its performance was evaluated as a function of coexistence ions using column adsorption.

During two seasons, we sampled stream water used as potable water in Fukushima prefecture. Table 1 shows the quality of the sampled stream water. The concentrations of $\mathrm{K}, \mathrm{Ca}, \mathrm{Mg}$, and $\mathrm{Na}$ were independent of season. Based on this information, we used $1 \mathrm{mg} / \mathrm{L}$ of coexistence ions in this study. Fig. 8 and Fig. 9 show the results of column adsorption measurements conducted on a $1 \mu \mathrm{g} / \mathrm{L} \mathrm{Cs} \mathrm{solu-}$ tion by adding $1 \mathrm{mg} / \mathrm{L}$ of $\mathrm{K}, \mathrm{Ca}, \mathrm{Mg}$, and $\mathrm{Na}$. The breakthrough point (BP) was defined as when the Cs concentra-

Table 1 Stream water sample from Fukushima prefecture. $[\mathrm{mg} / \mathrm{L}]$

\begin{tabular}{ccccc}
\hline \hline Month & $\mathrm{K}$ & $\mathrm{Ca}$ & $\mathrm{Mg}$ & $\mathrm{Na}$ \\
\hline May & $0.6-0.8$ & $1.3-3.7$ & $0.3-0.5$ & $3.3-5.5$ \\
\hline Nov. & $0.6-0.8$ & $1.4-3.7$ & $0.3-0.6$ & $3.8-5.9$ \\
\hline
\end{tabular}

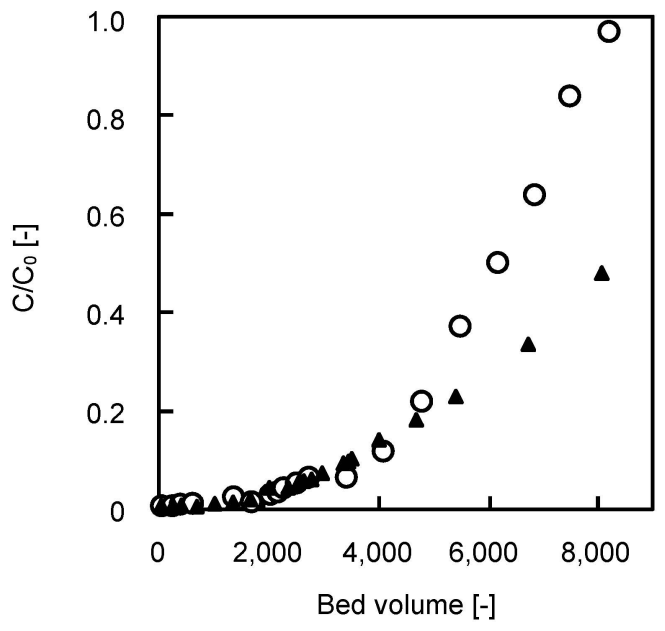

Fig. 8 Breakthrough curves with coexisting ion for $\mathrm{Dg}=200 \%$. The molar ratio of $9 \mathrm{G}$ to GMA is $1 \mathrm{~mol} \%$; $(\bigcirc)$ noncoexising ion, ( $\boldsymbol{\Delta}) 1 \mathrm{mg} / \mathrm{L}$ of coexisting ion.

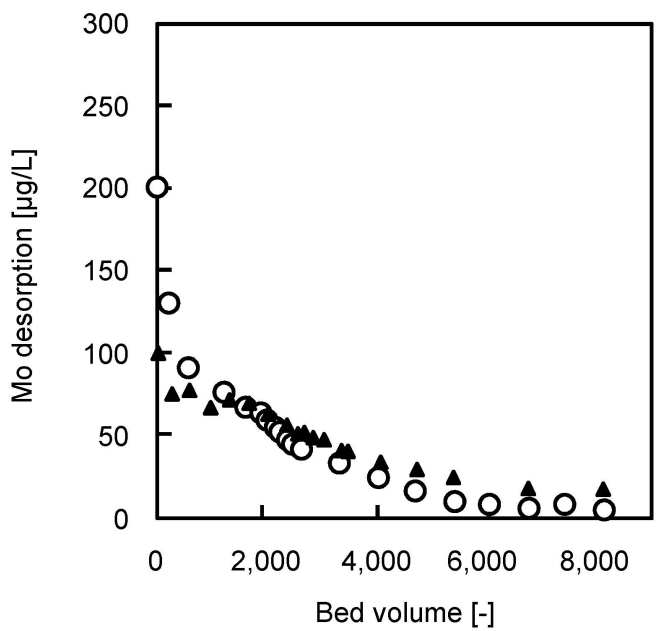

Fig. 9 Desorbed Mo with noncoexisting and coexisting ion for $\mathrm{Dg}=200 \%$. The molar ratio of $9 \mathrm{G}$ to GMA is $1 \mathrm{~mol} \%$; (O) noncoexisting ion, ( $\boldsymbol{\Delta}) 1 \mathrm{mg} / \mathrm{L}$ of coexisting ion. 
tion $C$ at the column outlet reached $5 \%$ of the feeding solution concentration $C_{0}$. The $\mathrm{BP}$ of $\mathrm{Cs}$ adsorption for the case of no coexisting ions and with coexisting ions was 2,650 and 2,630 bed volume (BV), respectively, as shown in Fig. 8. The efficiency of $\mathrm{Cs}$ adsorption had an insignificant influence of $1 \mathrm{mg} / \mathrm{L}$ on the coexisting ion at the BPs of the noncoexising ions $(1 \mathrm{mg} / \mathrm{L}$ of coexistence is essentially the same). Both $\mathrm{Cs}$ adsorption capacities at the $\mathrm{BP}$ were the same: $0.7 \mathrm{mmol}-\mathrm{Cs} /$ (kg-adsorbent). However, the degree of adsorption of $\mathrm{K}, \mathrm{Ca}, \mathrm{Mg}$, and $\mathrm{Na}$ was almost $0 \%$ for all BVs. Thus, the proposed grafted adsorbent has high selectivity for Cs. Fig. 9 shows the concentration of desorbed Mo as determined by column adsorption experiments. Previous results ${ }^{7)}$ showed that the concentration of desorbed Mo reached the order of $\mathrm{mg} / \mathrm{L}$. The proposed adsorbent was decreased to the $\mu \mathrm{g} / \mathrm{L}$ level by introducing the crosslinked structure into the adsorbent for attaining highly stable AMP.

\section{References}

1) Ministry of the Environment in Japan, "Results of Monitoring Streamwater (2014.3.26)". Jpn. (2014), press release 20.

2) Ministry of Health, Labour and Welfare in Japan, "Establishment of the limits for Radionuclide in Foods". (2014).

3) H. Mimura and I. Yamagishi, J. Ion. Exchange, 23 (2), 29-42 (2012).

4) Y. Okamura, K. Fujiwara, R. Ishihara, T. Sugo, T. Kojima, D. Umeno, and K. Saito, Radiat. Phys. Chem., 94, 119-122 (2014).

5) M. R. Awual, S. Suzuki, T. Taguchi, H. Shiwaku, Y. Okamoto, and T. Yaita. Chem. Eng. J., 242, 127-5 (2014).

6) T. A. Todd, N. R. Mann, T. R. Tranter, F. Šebesta, J. John, and A. Motl. J. Radioanal. Nucl. Chem., 254, 47-52 (2002).

7) A. Iwanade, N. Kasai, H. Hoshina, Y. Ueki, S. Saiki, and N. Seko. J Radioanal. Nucl. Chem., 293, 703-709 (2012). 